\title{
Delayed Diagnosis of Anorectal Malformations in Neonates
}

Running title: Anorectal malformation delayed diagnosis

Paul Kruger MD ${ }^{1,2}$, Warwick J. Teague DPhil FRACS ${ }^{1,2,3}$,

Rija Khanal MD ${ }^{1,2}$, John M. Hutson AO DSc FRACS ${ }^{2,3,4}$,

Sebastian K. King PhD FRACS ${ }^{1,2,3,5}$

${ }^{1}$ Department of Paediatric Surgery, The Royal Children's Hospital,

Melbourne, Victoria, Australia

${ }^{2}$ Surgical Research, Murdoch Children's Research Institute, Melbourne,

Australia

${ }^{3}$ Department of Paediatrics, University of Melbourne, Victoria, Australia

${ }^{4}$ Department of Urology, The Royal Children's Hospital, Melbourne, Victoria, Australia

${ }^{5}$ Department of Gastroenterology and Clinical Nutrition, The Royal Children's Hospital, Melbourne, Victoria, Australia

Correspondence to:

Associate Professor Sebastian King

Department of Paediatric Surgery

The Royal Children's Hospital

This is the author manuscript accepted for publication and has undergone full peer review but has not been through the copyediting, typesetting, pagination and proofreading process, which may lead to differences between this version and the Version of Record. Please cite this article as doi: 10.1111/ans.15374

This article is protected by copyright. All rights reserved. 
50 Flemington Road

Parkville, Melbourne, Victoria

Australia 3052

(P) +61 393455801

(F) +61393456668

(E) sebastian.king@rch.org.au

This study was presented at the Pacific Association of Pediatric Surgeons in Hawaii, April 2016.

One table

Abstract: 196 words

Text: 1212 words

This article is protected by copyright. All rights reserved. 


\section{Abstract}

\section{Background}

Anorectal malformations (ARM) are common congenital abnormalities of the terminal hindgut. Ideally, ARM should be diagnosed at, or shortly following, birth by careful physical examination of the perineum. Delayed diagnosis has been implicated as a risk factor for complications, including intestinal perforation. This study aimed to determine the rate of delayed diagnosis and associated intestinal perforation in ARM.

\section{$\underline{\text { Methods }}$}

A retrospective review was performed of all ARM patients managed at The Royal Children's Hospital over a 16-year period (2000 - 2015). Data collected included ARM type, timing of diagnosis, and complications. Delayed diagnosis was defined as being at more than 24 hours of age.

\section{$\underline{\text { Results }}$}

A total of 243 ARM patients (male 146/243, 60\%) were included. The most frequent ARM types were perineal fistula (83/243, 34\%) and rectovestibular fistula $(40 / 243,16 \%)$. Diagnosis was delayed beyond 24 hours of age in 92/243 (38\%) patients. The ARM type most commonly delayed in diagnosis 
was perineal fistula $(37 / 83,45 \%)$. Two patients in whom diagnosis was delayed suffered an intestinal perforation.

\section{Conclusion}

Delayed diagnosis in ARM patients remains a common, and potentially fatal, occurrence. Improved assessment of newborns is required to ensure timely diagnosis of ARM, and avoidance of complications associated with delayed diagnosis.

Keywords: neonate, anorectal malformation, delayed, perforation 


\section{Introduction}

Anorectal malformations (ARM) are relatively common congenital abnormalities of the terminal hindgut, which exist on a wide spectrum, ranging from a simple perineal fistula to a complex cloacal exstrophy. The incidence of ARM ranges from 1:2-5000 live births. ${ }^{1}$

Ideally ARM should be diagnosed at, or shortly following, birth by careful physical examination of the perineum. Patients with a delayed diagnosis of ARM may present with bowel obstruction, chronic constipation, and even intestinal perforation. ${ }^{2}$ Unfortunately, these presentations are not uncommon, even in first world centres. ${ }^{3-6}$ Complications arising from delayed diagnosis are significant, and may be life-threatening in cases of intestinal perforation. ${ }^{7}$ Mortality following intestinal perforation may exceed $50 \%$ in premature neonates, or neonates with associated anomalies. ${ }^{8}$ It has been suggested that reluctance to use rectal thermometers in neonates may have led to an increased rate of missed ARM. ${ }^{5}$

Clinicians must be thorough in their examination of neonates to ensure timely and accurate diagnosis of an ARM. By retrospectively analysing cases of ARM managed at our tertiary referral centre we aimed to determine the rate of delayed diagnosis amongst patients, and the risk of intestinal perforation. 


\section{Methods}

A retrospective review of all patients with an ARM managed at The Royal between January 2000 - November 2015 was performed. Patients were identified from the Neonatal Intensive Care Unit patient registry, the operating theatre databases and admissions coded by health information systems. All patients were transferred to the hospital for tertiary level care. Ethical approval was obtained from the institutional Hospital Research Ethics Committee (DA058-2014-01).

All medical records were reviewed to record: (1) specific subtype of ARM, (2) associated anomalies identified, and, (3) evidence of delayed diagnosis (defined by diagnosis of the ARM being made at more than 24 hours of life). Associated anomalies were recorded when detected on investigations or mentioned in consultation letters and/or discharge summaries. To ensure accuracy in determining the incidence of delayed diagnoses, patient records were used to identify the specific timing of ARM diagnosis and determine whether this was before or after 24 hours of life. This was achieved by analysing state-wide neonatal emergency retrieval service records, admission notes, and nursing notes.

Study data were collected and managed using REDCap electronic data capture tools hosted at our research institution. ${ }^{9}$ The definitive diagnoses were confirmed by the senior author. SPSS software (IBM Corp. Released 2013. IBM SPSS Statistics for Macintosh, Version 22.0, Version 22.0. 
Armonk, NY: IBM Corp) was used for subgroup analyses, including Fisher's exact test. Demographic data were described with frequencies and proportions.

This article is protected by copyright. All rights reserved. 


\section{Results}

A total of 243 patient records were reviewed, in which the majority $(146 / 243,60 \%)$ of patients was male. The most frequent types of ARM were perineal fistula $(83 / 243,34 \%)$, rectovestibular fistula $(40 / 243,16 \%)$ and rectoprostatic fistula $(26 / 243,11 \%)$. Sixty of the $243(25 \%)$ patients had no other documented congenital anomaly.

A delayed diagnosis occurred in $92 / 243$ (38\%) patients. The majority $(53 / 92,58 \%)$ of these patients were males. Patients with anal stenosis were the most likely to present with a delayed diagnosis $(17 / 25,68 \%)$, followed by other ARM subtypes limited to the perineum (covered anus, anal membrane, perineal fistula) (Table 1). In the 183 patients with a documented associated anomaly, the majority $(125 / 183,68 \%)$ were diagnosed before 24 hours of age. Conversely, in those patients with no other documented anomaly, the majority $(34 / 60,57 \%)$ were diagnosed after 24 hours of age $(p<0.001)$.

Two patients $(2 / 92,2 \%)$ in whom diagnosis was delayed suffered an intestinal perforation. One patient had a rectal atresia in the setting of Down syndrome and survived following colostomy formation. ${ }^{2}$ The other patient had a perineal fistula and survived following colostomy formation. No patients in whom the diagnosis of ARM was made before 24 hours of age suffered an intestinal perforation. Thus, the overall incidence of intestinal perforation in this cohort was $2 / 243(0.8 \%)$. 


\section{Discussion}

We have described a well-defined cohort of ARM patients, managed at our large tertiary referral centre over a 16-year period. In more than one-third of the patients, the initial diagnosis of the ARM was made after 24 hours of age. The delay in diagnosis was most often the consequence of a more distally placed anomaly, with over two-thirds of anal stenosis patients presenting late.

There is a body of literature that complements the findings in our study. The published incidences of delayed diagnosis range from 13\% in larger studies ${ }^{3-5,10-11}$ to $53 \%$ in a smaller study from Leicester, UK. ${ }^{6}$ Other authors have employed a definition of delayed diagnosis as being beyond 48 hours of age. ${ }^{3,5}$ However, evidence would suggest that the incidence of intestinal perforation increases significantly after 24 hours, and often occurs before 48 hours. ${ }^{3,8-9,12-14}$ Consequently, we defined a delay in diagnosis as more than 24 hours of age, a definition previously employed by Lindley et al. and Haider et al. ${ }^{6,10}$

Spontaneous intestinal perforation in ARM is a rare and highly morbid event, and a delay in ARM diagnosis is considered to increase the risk of this complication. ${ }^{3,10,13-15}$ Previous studies report an increased incidence of intestinal perforation in the setting of delayed ARM diagnosis, ranging from 3$9.6 \%$. Our incidence of two cases in a cohort of 92 patients (2\%) is lower than 
these reports, but consistent when one considers the impact of a single event within relatively small sample sizes.

The proposed aetiology for perforation in cases of ARM is distal occlusion, resulting in distension and ischaemia, progressing to gangrene and perforation. ${ }^{15}$ The rate of significant morbidity directly related to perforation in ARM may be as high as $57 \% .{ }^{13}$ Mortality among patients who suffer intestinal perforation may reach $50 \%$, particularly in neonates who are premature or have associated anomalies. ${ }^{8,14-15}$

Intuitively, those patients with subtypes of ARM that are less anatomically displaced are more likely to have a delayed diagnosis. Our findings of perineal fistula and anal stenosis being more commonly missed are consistent with this expectation and the literature. ${ }^{3}$ However, Lindley et al. found no significant differences in the subtypes of ARM between patients with a delayed diagnosis and those diagnosed appropriately. ${ }^{10}$

In our cohort, the ARM diagnosis was more likely to be delayed if there was no other associated anomaly detected. The detection of a significant congenital anomaly, either in the antenatal or early postnatal period, will often prompt clinicians to more accurately examine the perineum. ${ }^{11}$ However, even in those patients with an associated anomaly, the rate of delayed diagnosis was greater than $30 \%$.

A contributing factor to the ongoing inability worldwide to routinely diagnose ARM in a timely fashion may be the declining use of rectal 
thermometers. ${ }^{5}$ In addition, inconsistency in performing routine newborn screens, and the relative infrequency of ARM, may have led to clinicians lacking the required skills to accurately diagnose such a malformation.

Within the limits of a retrospective study, we have demonstrated the ongoing issue of delayed diagnosis in patients with ARM. As we were able to access all transfer data, and admission notes, we were able to determine the time to diagnosis with great accuracy. As all patients at out tertiary centre were born outside the hospital, we need to focus our education for the referring institutions upon the essential requirements of the newborn examination. 


\section{Acknowledgements}

Associate Professor Sebastian King and Associate Professor Warwick

Teague are both generously supported by The Royal Children's Hospital Foundation.

This article is protected by copyright. All rights reserved. 


\section{References}

[1] Nah SA, Ong CC, Lakshmi NK, et al. Anomalies associated with anorectal malformations according to the Krickenbeck anatomic classification. J Pediatr Surg 2012;47:2273-8.

[2] King SK, Cooksey R, Atkinson J, et al. Colonic perforation in a neonate with an anorectal malformation. ANZ J Surg 2014 DOI: 10.1111/ans.12704 [3] Turowski C, Dingemann J, Gillick J. Delayed diagnosis of imperforate anus: an unacceptable mortality. Pediatr Surg Int 2010;26:1083-6.

[4] Tareen F, Coyle D, Aworanti OM, Gillick J. Delayed diagnosis of anorectal malformation--a persistent problem. Ir Med J 2013;106:238-40.

[5] Wilson BE, Etheridge CE, Soundappan SV, et al. Delayed diagnosis of anorectal malformations: are current guidelines sufficient? J Paed Child Health 2010;46:268-72.

[6] Haider N, Fisher R. Mortality and morbidity associated with late diagnosis of anorectal malformations in children. The Surgeon 2007;5:327-30. [7] Tong WD, Ludwig KA. Neonatal colon perforation due to anorectal malformations: can it be avoided? World J Gastro 2013;19:3915-7.

[8] Digray NC, Mengi Y, Goswamy HL, et al. Colorectal perforations in neonates with anorectal malformations. Pediatr Surg Int 2001;17:42-4. [9] Harris PA, Taylor R, Thielke R, et al. Research electronic data capture (REDCap) - a metadata-driven methodology and workflow process for 
providing translational research informatics support. J Biomed Inform 2009;42:377-81.

[10] Lindley RM, Shawis RN, Roberts JP. Delays in the diagnosis of anorectal malformations are common and significantly increase serious early complications. Acta Paediatrica 2006;95:364-8.

[11] Kim HLN, Gow KW, Penner JG, et al. Presentation of low anorectal malformations beyond the neonatal period. Pediatrics 2000;105:e68-70. [12] Chan KW, Lee KH, Tsui SY, et al. Bowel perforation in newborn with anorectal malformation and no fistula at presentation. J Pediatr Surg 2014;49:390-4.

[13] Sandlas G, Kothari P, Sarda D, et al. Spontaneous bowel perforation in a neonate with anorectal malformation. Saudi J Gastro 2011;17:72-3.

[14] Raveenthiran V. Spontaneous perforation of the colon and rectum complicating anorectal malformations in neonates. J Pediatr Surg 2012;47:720-6.

[15] Eltayeb AA. Anorectal malformations presenting with colorectal perforation: frequency, associated morbidity and mortality. Surgical Practice 2014;18:23-6. 
This article is protected by copyright. All rights reserved. 
Table 1: Delayed diagnosis of ARM according to type $(n=243)$

\begin{tabular}{llll}
\hline ARM Type & Delayed & Total & Percentage \\
\hline Anal stenosis & 17 & 25 & 68 \\
Other & 6 & 12 & 50 \\
Perineal fistula & 37 & 83 & 45 \\
Rectal Atresia & 5 & 13 & 38 \\
Rectovesical / Bladderneck fistula & 3 & 8 & 38 \\
Rectovestibular fistula & 15 & 40 & 38 \\
Rectobulbar fistula & 2 & 8 & 25 \\
Rectovaginal fistula & 1 & 5 & 20 \\
\hline Rectoprostatic fistula & 5 & 26 & 19 \\
\hline Unknown type & 1 & 7 & 14 \\
\hline Cloaca: <3cm common channel & 0 & 7 & 0 \\
\hline Cloacal exstrophy & 0 & 7 & 0 \\
\hline Rectourethral fistula - & 0 & 2 & 0 \\
\hline unknown location & & & \\
\hline
\end{tabular}

"Other" - anal web, covered anus, duplication cyst, H-type fistula, mucosal tract (x 2), perineal fistula with urogenital sinus 


\section{University Library}

\section{- M M N E R VA A gateway to Melbourne's research publications}

Minerva Access is the Institutional Repository of The University of Melbourne

Author/s:

Kruger, P;Teague, WJ;Khanal, R;Hutson, JM;King, SK

Title:

Delayed diagnosis of anorectal malformations in neonates

Date:

2019-08-26

Citation:

Kruger, P., Teague, W. J., Khanal, R., Hutson, J. M. \& King, S. K. (2019). Delayed

diagnosis of anorectal malformations in neonates. ANZ JOURNAL OF SURGERY, 89 (10), pp.1253-1255. https://doi.org/10.1111/ans.15374.

Persistent Link:

http://hdl.handle.net/11343/286330 